\title{
1 Towards resolution of the intron retention paradox in breast cancer
}

2 Jaynish S. Shah ${ }^{1,2,3}$, Michael Milevskiy ${ }^{4}$, Veronika Petrova ${ }^{1,2}$, Amy YM Au ${ }^{2}$, Justin J.L.

3 Wong ${ }^{5,6}$, Jane E. Visvader ${ }^{4,7}$, Ulf Schmitz ${ }^{1,2,8^{*} \dagger}$, John E.J. Rasko ${ }^{2,6,9^{*} \dagger}$

$5{ }^{1}$ Computational BioMedicine Laboratory Centenary Institute, The University of Sydney,

6 Australia

$7{ }^{2}$ Gene \& Stem Cell Therapy Program Centenary Institute, The University of Sydney, 8 Australia

$9{ }^{3}$ Australian Centre for Blood Diseases, Central Clinical School, Monash University and 10 Alfred Health, Melbourne, VIC, Australia

$11{ }^{4}$ ACRF Cancer Biology and Stem Cells Division, The Walter and Eliza Hall Institute of

12 Medical Research, Parkville, VIC 3052, Australia

135 Epigenetics and RNA Biology Program Centenary Institute, The University of Sydney,

14 Camperdown 2050, Australia

$15{ }^{6}$ Faculty of Medicine \& Health, The University of Sydney, Australia

$16{ }^{7}$ Department of Medical Biology, The University of Melbourne, Parkville, VIC 3010,

17 Australia

$18{ }^{8}$ College of Public Health, Medical and Veterinary Sciences, Department of Molecular and

19 Cell Biology, James Cook University, Townsville, QLD 4811, Australia

$20 \quad{ }^{9}$ Cell and Molecular Therapies, Royal Prince Alfred Hospital, Camperdown, Australia

21 Therapies, Royal Prince Alfred Hospital, Camperdown, Australia

24 * To whom correspondence should be addressed:

25 Prof. John Rasko, email: j.rasko@centenary.org.au or A/Prof. Ulf Schmitz; email:

26 ulf.schmitz@jcu.edu.au

$27{ }^{\dagger}$ These authors should be regarded as joint last authors. 


\section{Abstract}

29 After many years of neglect in the field of alternative splicing, the importance of intron 30 retention (IR) in cancer has come into focus following landmark discoveries of aberrant IR

31 patterns in cancer. Many solid and liquid tumours are associated with drastic increases in IR 32 and such patterns have been pursued as both biomarkers and therapeutic targets.

33 Paradoxically, breast cancer $(\mathrm{BrCa})$ is the only tumour type in which IR is reduced compared 34 to adjacent normal breast tissue.

35 In this study, we have conducted a pan-cancer analysis of IR with emphasis on BrCa and its 36 subtypes. We explored mechanisms that could cause aberrant and pathological IR and 37 clarified why normal breast tissue has unusually high IR.

38 Strikingly, we found that reduced IR in $\mathrm{BrCa}$ can be largely attributed to normal breast tissue 39 having the highest occurrence of IR events compared to other healthy tissues. Our analyses 40 suggest that low numbers of IR events in breast tumours are associated with poor prognosis, 41 particularly in the luminal B subtype. Interestingly, we found that IR frequencies negatively 42 correlate with cell proliferation in $\mathrm{BrCa}$ cells, i.e. rapidly dividing tumour cells have the 43 lowest number of IR events. Aberrant RNA binding protein (RBP) expression and changes in 44 tissue composition are among the causes of low IR in BrCa.

45 Our results suggest that IR should be considered for therapeutic manipulation in $\mathrm{BrCa}$ 46 patients with aberrantly low IR levels and that further work is needed to understand the cause 47 and impact of high IR in other tumour types.

49 Keywords: alternative splicing, patient stratification, luminal B breast cancer, adipocytes, 50 cancer transcriptomics 


\section{Introduction}

52 Pre-mRNA splicing is a ubiquitous process that is crucial for the maintenance of

53 transcriptomic complexity and gene expression regulation in eukaryotic cells ${ }^{1,2}$. Perturbations

54 to this highly calibrated system can have severe consequences and lead to diseases including

55 cancer $^{3-6}$. In this context, numerous studies describing intron retention (IR) in disease have

56 shed light on the mechanisms leading to aberrant and pathological $\mathrm{IR}^{7-9}$.

57 The importance of IR in cancer has been emphasised following landmark discoveries about

58 (i) aberrant IR patterns in leukemia ${ }^{10,11}$, (ii) IR as a source of neoepitopes ${ }^{12}$, (iii) tumour

59 suppressor gene inactivation by intronic polyadenylation ${ }^{13}$, (iv) IR-based biomarkers ${ }^{14,15}$, and

60 (v) IR as a therapeutic target ${ }^{16}$.

61 IR is regulated by $c i s$ - and trans-acting modulators ${ }^{2,17,18}$ facilitating cellular responses to a

62 range of environmental stimuli ${ }^{19}$. Intron-retaining mRNA transcripts are often degraded via

63 nonsense-mediated decay (NMD), thereby causing down-regulation of the host gene. The

64 burden of IR in disease is governed by perturbations to mechanisms known to regulate this

65 form of alternative splicing, including mutations, splicing factor dysregulation, and

66 epigenetic variations.

67 However, despite the cumulative evidence for the importance of IR in cancer, a systematic

68 analysis of IR regulation in $\mathrm{BrCa}$ and the role of aberrant IR in $\mathrm{BrCa}$ biology has not been

69 conducted to date. In this study we sought to resolve the paradox wherein breast cancer

70 exhibits reduced IR, which is an important consequence of alternative splicing.

71 We analysed $615 \mathrm{BrCa}$ patient transcriptomes which included four major molecular subtypes

72 (Luminal A, Luminal B, Basal, and Her2 positive). We confirmed a consistent

73 downregulation of IR in BrCa. However, we also observed that normal breast tissue has a

74 significantly higher IR event frequency compared to other healthy tissues. The number of IR

75 events correlated with survival in the luminal B BrCa subtype. Differences in IR frequencies

76 are largely influenced by the tissue's cellular composition as well as specific dysregulated

77 RNA binding proteins (RBPs).

78 Results

79 High IR is associated with improved survival in Luminal B subtype breast cancer

80 To compare IR profiles across human cancers we retrieved transcriptomics data for nine

81 different solid tumours and matched adjacent normal tissues from The Cancer Genome Atlas 
82 (TCGA; Figure 1A) and quantified IR using the IRFinder algorithm, which we have 83 previously validated ${ }^{17}$. Overall, we identified a total of 11,943 unique IR events

84 (Supplementary Table 1), of which 917 were shared among all nine cancers analysed.

85 Our analyses confirmed a previous report that $\mathrm{BrCa}$ is the only cancer in which the number of

86 IR events is reduced compared to normal adjacent tissue (Figure 1B) ${ }^{20}$. All other cancers

87 exhibit increased IR compared to their matched adjacent normal tissue (Figure 1B). However,

88 we also noticed that the number of IR events in breast cancer itself was comparable with

89 other cancers, while normal breast tissue presented with unusually high numbers of IR events

90 (Figure 1B). In fact, normal breast tissue had the highest IR frequencies compared to all other

91 normal tissues (Figure 1C). Therefore, reduced IR in tumours, which is unique to $\mathrm{BrCa}$, can

92 be largely attributed to normal breast tissue having the highest occurrence of IR events.

93 We applied beta regression models to identify differentially retained introns (dIRs) and found

943,024 dIRs between normal and breast cancer (Supplementary Figure 1A). Of these 210 were

95 downregulated (in 160 genes) and 69 were upregulated (in 52 genes) with a $\geq 10 \%$ difference

96 in the IR ratio $(\Delta \mathrm{IR} \geq 0.1)$. Downregulated IR events in $\mathrm{BrCa}$ are associated with processes

97 related to cell cycle, nuclear division, and DNA replication among others (Supplementary

98 Figure 1B).

99 Next, we explored whether the specific pattern of IR in $\mathrm{BrCa}$ is associated with clinical

100 features. As shown in Supplementary Figure 2A and Figure 1D, IR patterns were distinct

101 between $\mathrm{BrCa}$ vs normal tissues as well as estrogen receptors positive (ER $\left.{ }^{+}\right)$vs negative (ER)

102 samples, respectively. The human epidermal growth factor receptor 2 (HER2) amplified

103 molecular subtype had the lowest average number of IR events $(n=1,731)$ compared to the

104 other three main subtypes Luminal A $(n=2,089)$, Luminal B $(n=2,113)$, and Basal $(n=$

105 2,018) (Figure 1E). Strikingly, HER2-amplified tumours are associated with a 2.9-fold

106 increased hazard ratio ( $p=0.001$, Supplementary Figure 2B). Moreover, advanced stage

107 tumours (Stage III) had the lowest average number of IR events $(n=1,913)$ compared to

108 Stage II $(n=2,064)$ and Stage I $(n=2,210)$ tumours (Figure 1F). Likewise, those tumours

109 with the highest immunohistochemical (IHC) staining score for HER2 (score: 3) exhibited

110 the lowest average number of IR events $(n=1,825)$ compared to score $1(n=2,095)$ or score

$1112(n=2,137)$ tumours (Supplementary Figure 2C). We also found that a high number of IR

112 events is associated with better survival in patients with the Luminal B subtype (Figure 1G;

113 Supplementary Figure 2D). Overall, these data support the conclusion that poor outcomes in

114 BrCa correlate with low IR. 
A

\begin{tabular}{|c|c|c|c|}
\hline $\begin{array}{l}\text { TCGA } \\
\text { Code }\end{array}$ & Cancer type & $\begin{array}{l}\text { matched } \\
\text { pairs* }(n)\end{array}$ & Total (n) \\
\hline$\overline{B L C A}$ & $\begin{array}{l}\text { Bladder urothelial } \\
\text { carcinoma }\end{array}$ & 14 & 28 \\
\hline BRCA & Breast invasive carcinoma & 84 & $\begin{array}{c}615 \\
(90 / 525)^{\prime}\end{array}$ \\
\hline COAD & Colon adenocarcinoma & 20 & 40 \\
\hline KIRC & $\begin{array}{l}\text { Kidney renal clear cell } \\
\text { carcinoma }\end{array}$ & 47 & 94 \\
\hline LIHC & $\begin{array}{l}\text { Liver hepatocellular } \\
\text { carcinoma }\end{array}$ & 31 & 62 \\
\hline LUAD & Lung adenocarcinoma & 14 & 28 \\
\hline LUSC & $\begin{array}{l}\text { Lung squamous cell } \\
\text { carcinoma }\end{array}$ & 39 & 78 \\
\hline PRAD & Prostate adenocarcinoma & 49 & 98 \\
\hline STAD & Stomach adenocarcinoma & 14 & 28 \\
\hline
\end{tabular}

B

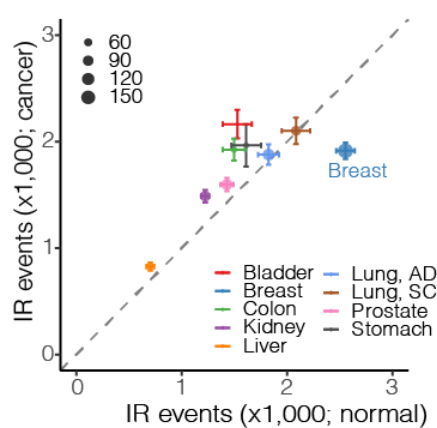

D

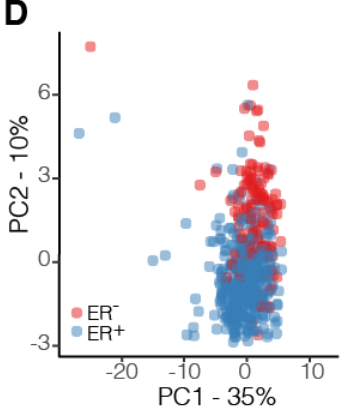

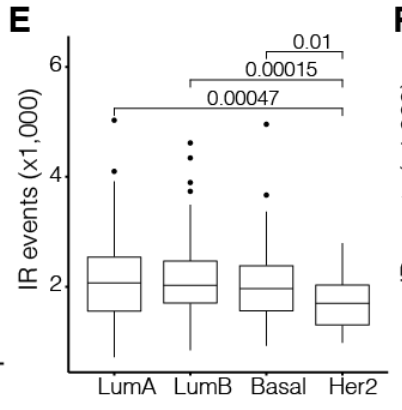

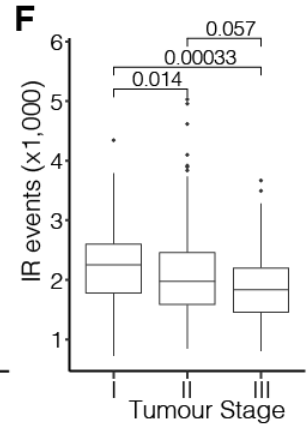

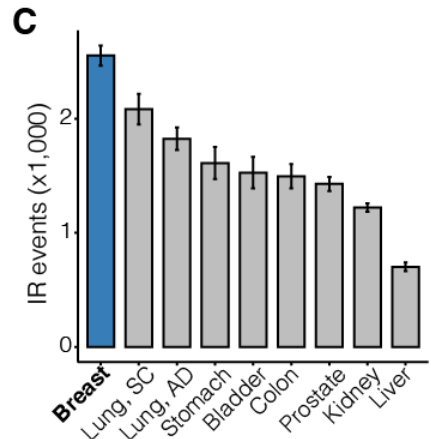

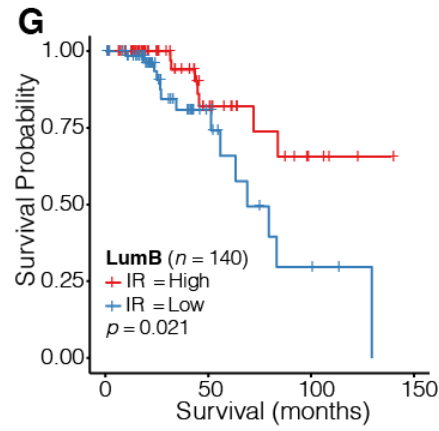

116 Figure 1 Breast cancer intron retention in the TCGA cohort. (A) Overview of TCGA samples analysed. (B)

117 Scatter plot illustrating the average number of IR events in cancers vs adjacent normal tissues. Error bars 118 indicate standard deviations. The size of the dots is proportional to the number of samples analysed. (C) Bar plot 119 showing the average number of IR events in normal tissues in descending order. Error bars represent standard 120 error of the mean. (D) PCA plot showing clusters of specific IR profiles in ER positive and ER negative tumour samples $(n=509)$. (E) Distributions of IR event frequencies in four major BrCa subtypes (LumA - Luminal A LumB -Luminal B; Basal, HER2 - human epidermal growth factor receptor 2 positive). (F) Distributions of IR event frequencies in tumour samples assigned to three tumour stages. (G) Kaplan-Meier plot illustrating the survival probabilities of Luminal B BrCa patients stratified by a high vs low number of IR events. Samples have been dichotomised based on the median number of IR events.

\section{Putative trans-regulators of IR in breast cancer}

128 We confirmed that IR decreases in BrCa cells in vitro based on our analysis of cultured $\mathrm{ER}^{+}$

129 MCF7 cells and non-tumorigenic MCF10A cells (Figure 2A). Higher IR in a normal breast epithelial cell line (MCF10A) was associated with reduced gene expression (Supplementary

131 Figure 3A).

132 To identify potential regulators of IR we correlated IR frequencies in the TCGA-BRCA 133 cohort with expression values (normalized RNA-seq counts) of $\sim 23,000$ genes

134 (Supplementary Table 2). We performed Gene Ontology (GO) enrichment analysis on the top $1355 \%$ genes with the highest $(r>0.41)$ and lowest $(r<-0.27)$ correlation coefficients to identify 136 potential positive and negative regulators of IR, respectively. We found eight GO terms that 137 were significantly enriched in positively correlated genes ( $p$-adj. $\leq 0.05$; Figure 2B). 138 Intriguingly, six of these GO terms were related to RNA splicing, with $D D X 39 B, R B M 25$, 
bioRxiv preprint doi: https://doi.org/10.1101/2022.03.04.482791; this version posted March 5, 2022. The copyright holder for this preprint (which was not certified by peer review) is the author/funder, who has granted bioRxiv a license to display the preprint in perpetuity. It is made available under aCC-BY-NC-ND 4.0 International license.

139 PRPF39, and SRSF11 being among the genes that most highly correlate with the number of

140 IR events in each sample (Figure 2C).

141 The four genes that most strongly anti-correlate with the number of IR events include the 142 mutase PGAM1, the membrane protein encoding SURF4, the mitochondrial transmembrane 143 transporter SLC25A5, and the mitochondrial ATP Synthase F1 Subunit Beta (ATP5F1B) 144 (Figure 2D). Strikingly, the top 10 most significant GO terms (out of 399) associated with 145 genes that negatively correlate with the number of IR events correspond to mitochondrial 146 processes and cellular energetics (Supplementary Figure 3B).

A
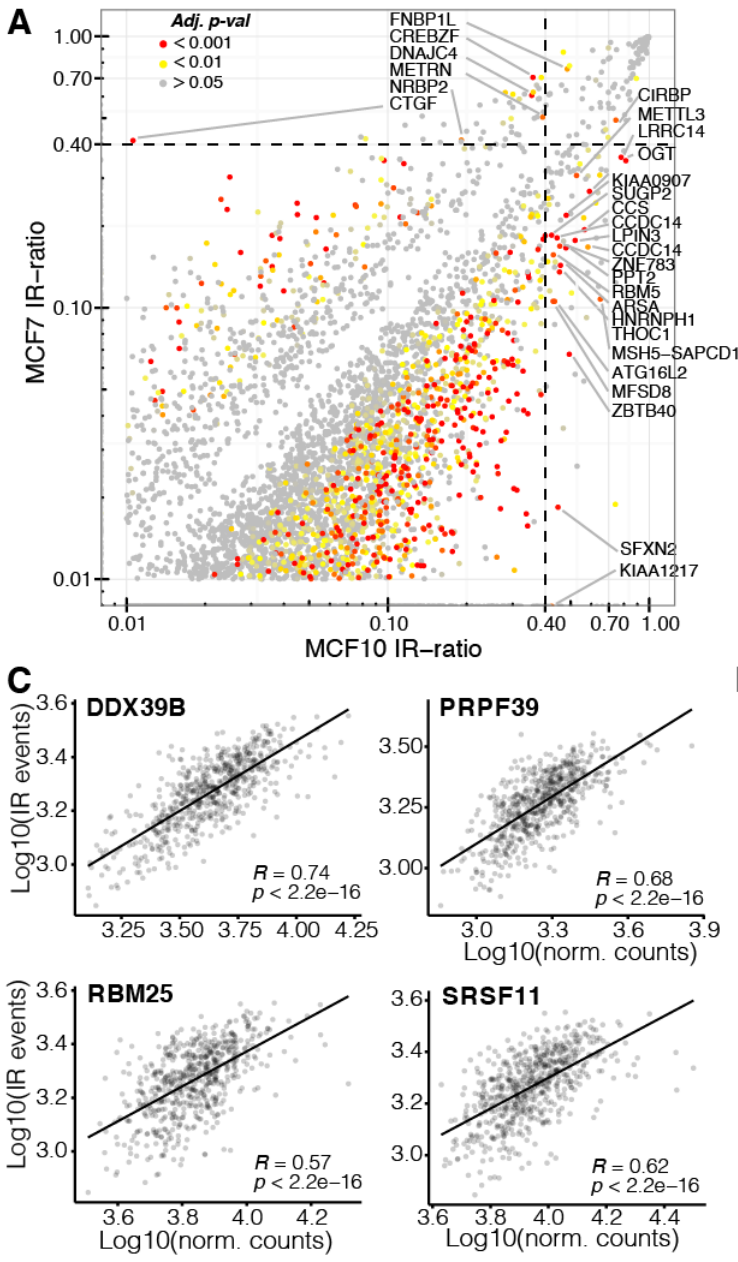

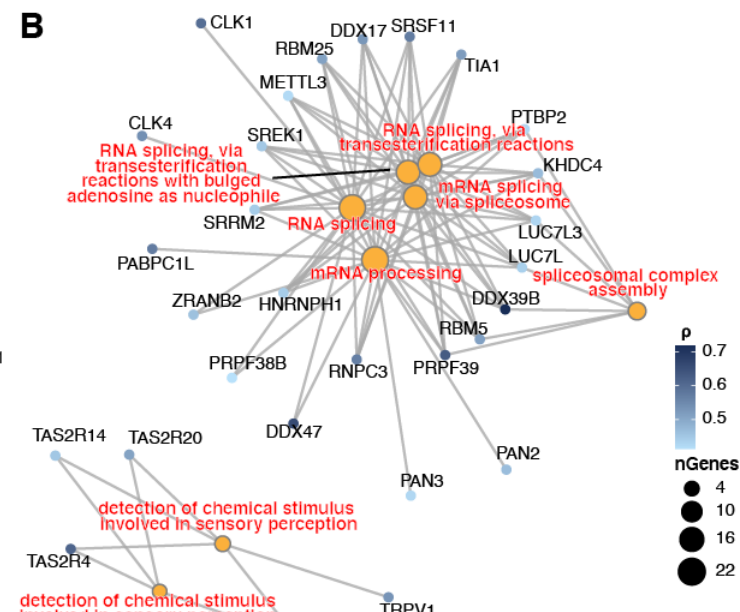

Figure 2 Putative regulators of intron retention in breast cancer. (A) Scatterplot showing differentially retained introns (dIR) between MCF7 and MCF10A cells. Genes associated with significant dIR events (p-adj. $0.001)$ and IR-ratios $>0.4$ are labelled. Given the low number of replicates $(n=2)$ we have applied the Audic and Claverie test $\mathrm{t}^{21}$ for significance testing. (B) Enriched GO terms in genes positively correlated with IR. Circle size of the GO terms (orange) is denoted by the number of genes associated with them. (C) RNA splicing associated genes that most highly correlate with the number of IR events in each sample. The scatterplots illustrate the $\log 10$ number of IR events against the $\log 10$ normalized read counts for each gene. (D) Genes that most strongly anti-correlate with the number of IR events in each sample. 


\section{Highly proliferating cells have fewer IR events}

158 Since the energy demand of a cell is tightly coupled with proliferation, we sought to examine

a potential link between doubling times of $\mathrm{BrCa}$ cells and the number of IR events in 36

160 BrCa-related cell lines of the Cancer Cell Line Encyclopedia (CCLE). Indeed, we found that

161 cells with slower doubling time exhibit a higher number of IR events (Figure 3A).

162 To corroborate this result, we also investigated whether a common proliferation marker 163 would inversely correlate with IR frequencies. Since immunohistochemistry (IHC) staining 164 of Ki-67 is unavailable for the TCGA cohort, we tested whether the proliferation rate in 165 tissues might be estimated based on MKI67 sequencing read counts. The MKI67 gene 166 encodes the proliferation marker protein Ki-67. Using Human Protein Atlas data, we confirmed that MKI67 mRNA expression correlates with its Ki-67 protein staining intensities detected by IHC (Supplementary Figure 4A). As expected, normalised MKI67 read counts were higher in all nine cancers when compared to the respective adjacent normal tissues

170 (Supplementary Figure 4B). This suggests that MKI67 read counts can be used as proxy for 171 IHC staining to estimate cellular proliferation rates. MKI67 expression was also found to be 172 inversely correlated to the doubling time of 36 CCLE cell lines (Supplementary Figure 4C). 173 We observed that the number of IR events in samples of the TCGA-BRCA cohort negatively 174 correlated with the proliferation rate (Figure 3B).
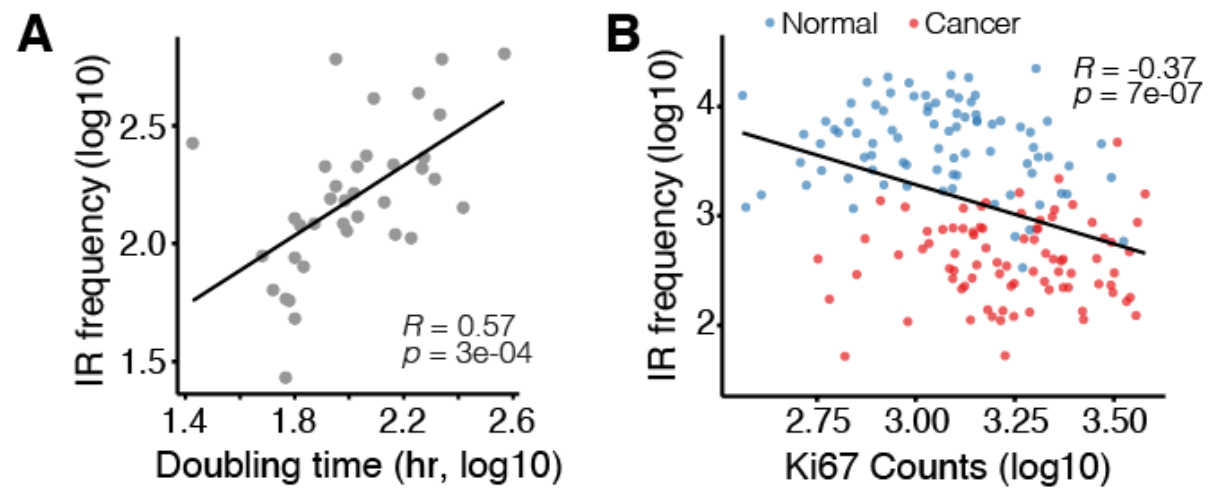

Figure 3 IR and cell proliferation. (A) Cancer Cell Line Encyclopedia (CCLE) cell doubling times (x-axis) correlate with number of IR events (y-axis). (B) Normalized read counts of proliferation marker Ki67 anticorrelate with the number of IR events (y-axis). Red dots - tumour samples; blue dots - normal breast tissue.

181 Next, we investigated genes that were specifically deregulated in $\mathrm{BrCa}$. We identified a set of 182150 genes that were only differentially expressed between $\mathrm{BrCa}$ and normal breast tissues, of 
183 which seven were RBPs (Figure 4A). We calculated the $z$-score of each gene's log fold-

184 change in $\mathrm{BrCa}$ versus the log fold-change in other cancers in order to estimate the level of 185 specificity of a gene being differentially expressed in $\mathrm{BrCa}$ only (Figure 4B). Among the

186 genes that are highly specifically over-expressed in BrCa are two known RBPs: ZFP36L2 187 and TUT4. ZFP36L2 promotes poly(A) tail removal of mRNA transcripts ${ }^{22}$, while Terminal 188 Uridylyl Transferase 4 (TUT4) adds uridines to deadenylated transcripts ${ }^{23}$. Thus, both RBPs 189 are mediators of mRNA decay, which could explain the observed reduction of IR transcripts 190 in $\mathrm{BrCa}$.

191 We also determined the frequency by which BrCa-specific genes occur in RNA-related gene 192 sets $(n=138)$ in the Molecular Signatures Database (MSigDB; total 5,000 curated gene 193 sets) ${ }^{24}$. While known RBPs such as ZFP36L2 and SNRNP25 (part of the minor U12-type 194 spliceosome) are annotated in multiple RNA-related gene sets, other genes, that are 195 specifically differentially expressed in $\mathrm{BrCa}$, did not show any potential RNA binding 196 capabilities (Supplementary Figure 5A).

197 In addition, we analysed differentially retained introns for occurrences of RBP binding 198 motifs. We found that differentially retained introns were enriched in NELFE and SRSF9 199 binding sites in upstream exons (5'-up) and the 3' terminal region, respectively (Figure 4C). 200 Moreover, retained introns have fewer HNRNPH1 and HNRNPK binding sites in their 3' 201 terminal region compared to non-retained introns (Figure 4C; Supplementary Figure 5B).

202 We conclude, that RPBs are among the factors that facilitate reduced IR in BrCa by enabling 203 efficient splicing of introns from pre-mRNA transcripts. However, RBPs specifically 204 differentially expressed in $\mathrm{BrCa}$ are not among those with enriched binding motifs within and 205 around differentially retained introns. This suggests that more complex, multifaceted 206 regulatory mechnisms are causing the consistent reduction of IR in $\mathrm{BrCa}$. 
bioRxiv preprint doi: https://doi.org/10.1101/2022.03.04.482791; this version posted March 5, 2022. The copyright holder for this preprint (which was not certified by peer review) is the author/funder, who has granted bioRxiv a license to display the preprint in perpetuity. It is made available under aCC-BY-NC-ND 4.0 International license.
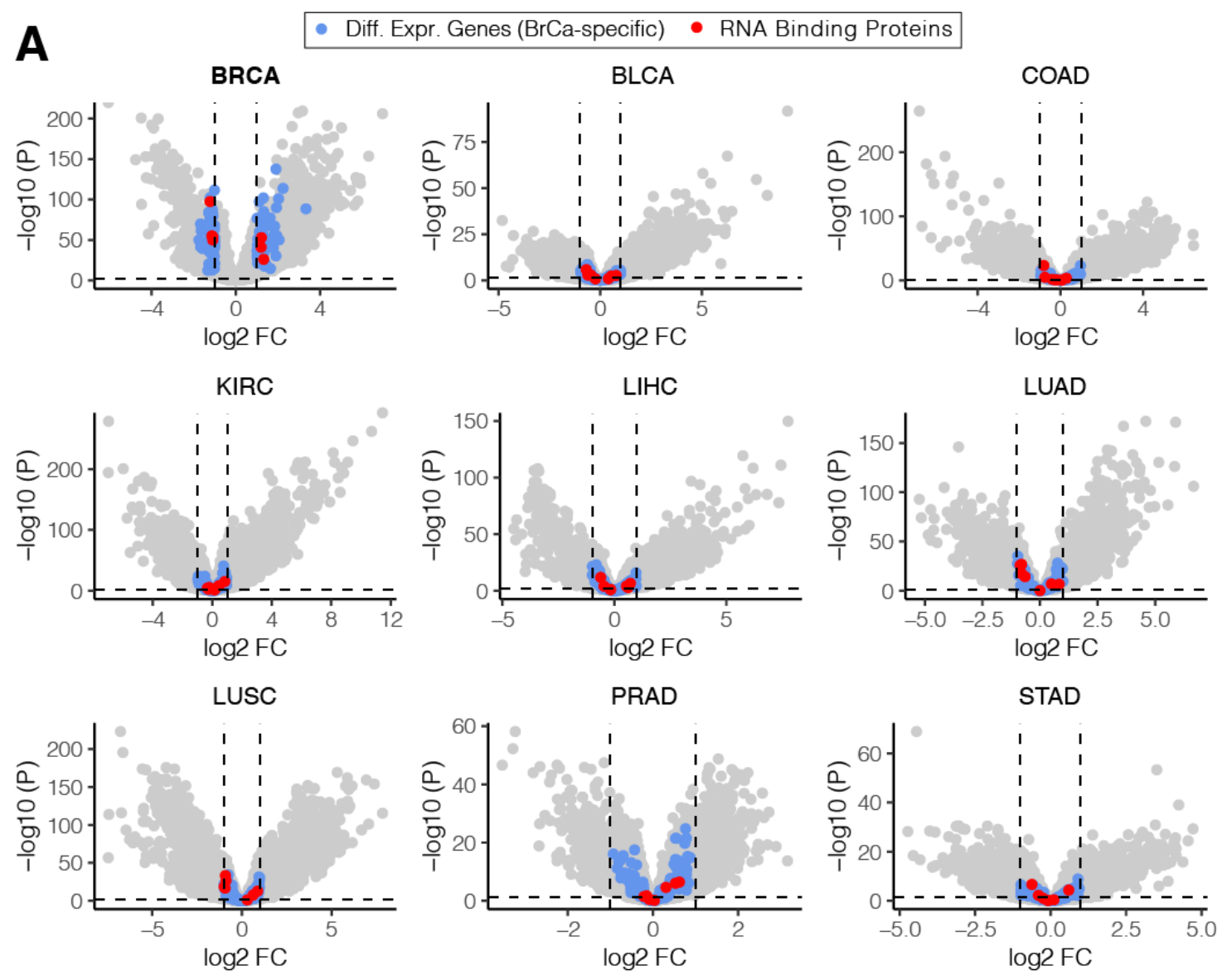

B
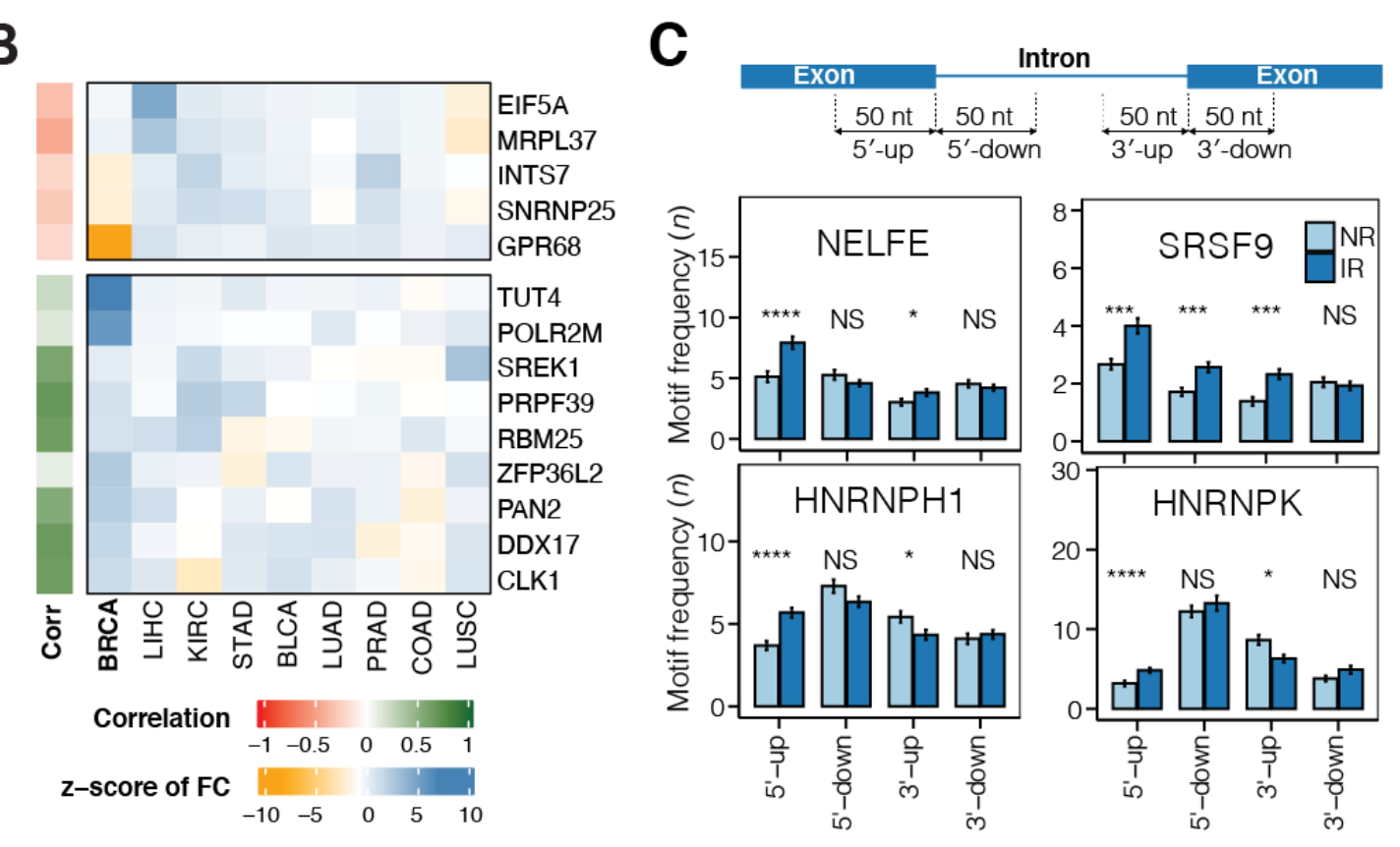

Figure 4 Breast cancer-specific gene expression and RBP analysis. (A) Volcano plots showing differentially expressed genes in nine tumour types vs adjacent healthy tissue. The dashed lines represent the $p$-value cut-off (horizontal; $p<0.05$ ) and fold-change threshold (vertical $|F C| \geq 1$ ). See Figure 1A for cancer type

211 abbreviations. Highlighted in blue are genes that are exclusively differentially expressed in $\mathrm{BrCa}$, while those in red represent RBPs within this subset. (B) Heatmap of genes specifically differently expressed in $\mathrm{BrCa}$ 
(represented by colour-coded z-score). Annotation bar (left) shows the colour-coded correlation coefficient between gene expression and number of IR events in each sample. (C) Bar plots show the frequencies with which known binding motifs occur around the splice sites (50 nt up-/downstream) of differentially retained (IR; dark blue) and non-differentially retained introns (NR; light blue). Differences in average frequencies were determined using students $t$-test. $* p<0.05, * * * p<0.001, * * * *<<0.0001, \mathrm{NS}-$ not significant.

\section{Tissue composition affects cancer IR profiles}

220 Since the reduction in IR events in $\mathrm{BrCa}$ contrasts with all other cancer types analysed, we 221 examined a possible contribution from the changing cell composition in the tumour 222 microenvironment compared to healthy breast tissue. Gene signature-based and machine 223 learning-based algorithms have been developed to deconvolute the cell type composition in 224 bulk RNA-sequencing data ${ }^{25}$. To compare cell environmental profiles of TCGA breast tumour samples versus healthy adjacent control samples we used the cell type deconvolution algorithm $\mathrm{xCell}^{26}$, which was trained on 1,822 pure human cell type-specific transcriptomes extracted from single cell transcriptome profiling data. xCell analysis revealed that the breast tumour cell composition is distinct from other cancers (Supplementary Figure 6). Among the most enriched cell types in $\mathrm{BrCa}$ are T-helper cells, mesenchymal stem cells and basophils.

230 These predictions are supported by recent single-cell BrCa profiling studies ${ }^{27-29}$. Normal 231 breast tissue is enriched in endothelial cells, adipocytes, and dendritic cells (Figure 5A).

232 Indeed, adipocyte and myeloid cell (M1 macrophages, basophils) enrichment is specific to 233 normal breast tissue (Figure 5B/C), which could explain the IR paradox in BrCa.

234 To determine whether cell types enriched in normal breast tissue have particularly high IR 235 event frequencies we retrieved RNA sequencing data of 66 cell/tissue types from the 236 ENCODE repository (Supplementary Table 3). Our analysis suggests that breast epithelial 237 cells have the highest prevalence of IR followed by adipocytes (Figure 5C), which could 238 explain the drop in IR events in breast tumours. 
A

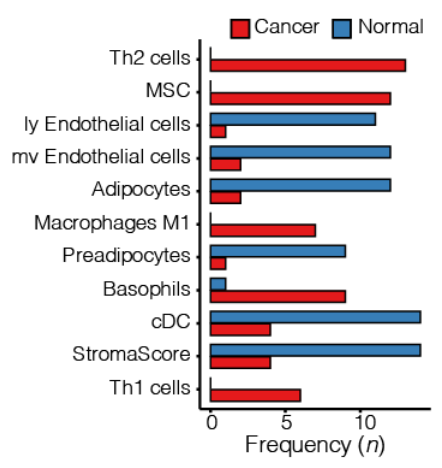

B

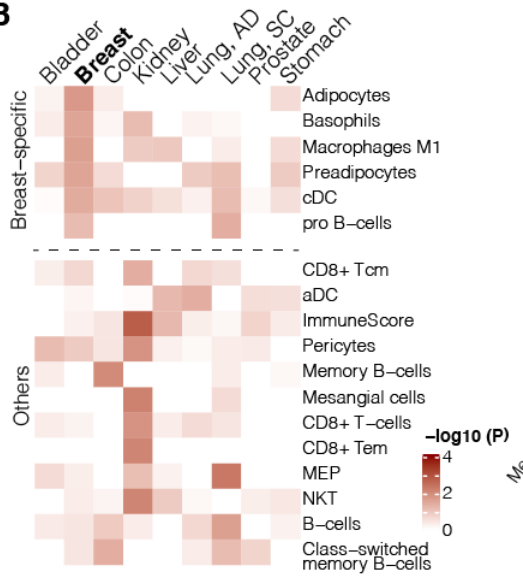

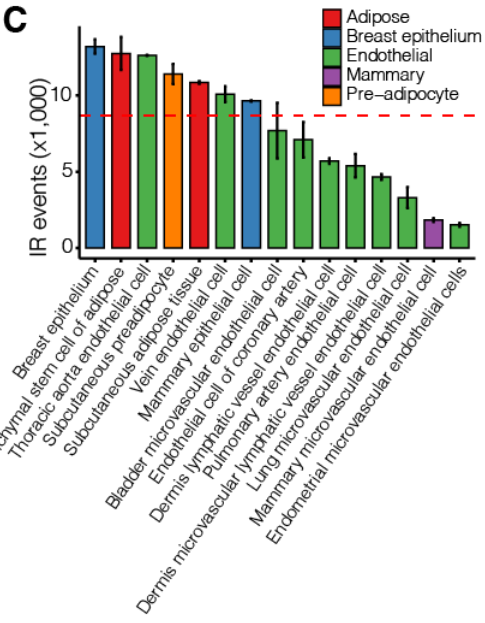

240

241

242

243

244

245

246

247

248

249

250

251

252

253

254

255

256

257

258

259

260

261

262

263

264

265

Figure 5 Breast tumour cell composition. (A) Frequently enriched cell types in breast tumours (red) and normal breast tissue (blue). (B) Heatmap illustrating cell type enrichment in healthy adjacent tissue of nine TCGA cancer cohorts. (C) Abundance of IR events in purified cells. Colours indicate groups of cells belonging to the same family. Dashed red line represent mean number of IR events. Th1/2 - T helper $1 / 2$; MSC mesenchymal stem cell; ly - lymphatic; mv - microvascular; a/cDC - activated/classical dendritic cell; Tcm - T central memory cell; Tem - T effector memory cell; NKT - natural killer T cell; MEP - Megakaryocyteerythroid progenitor cell. ImmuneScore quantifies the enrichment of an immune cell signature including B-cells, T-cells, DC, eosinophils, macrophages, monocytes, mast cells, neutrophils, and NK cells. StromaScore quantifies the enrichment of a stroma-type cell signature including adipocytes, endothelial cells, and fibroblasts.

\section{Discussion}

IR is omnipresent in vertebrate species ${ }^{2,30}$ and affects up to $80 \%$ of human protein-coding genes ${ }^{17}$. Numerous studies have highlighted the functional importance of retained introns in a wide range of biological functions including cell differentiation and development ${ }^{12,31-34}$.

Since first reports in 2015 and subsequent confirmatory studies, BrCa has stood in stark contrast to other cancers in relation to its burden of $\mathrm{IR}^{20}$. Dysregulation of cis- and transmodulators can cause aberrant IR in various cancers ${ }^{20}$. For example, Dvinge et al. found that snRNA expression changes IR in the MCF7 cell line and to a certain degree in BrCa patient samples. They also showed that splicing factor knockdown can lead to increased IR in triplenegative $\mathrm{BrCa}(\mathrm{TNBC})^{35}$. Kim et al. found that some BrCa IR events anti-correlate with DNA methylation and that high IR levels in transcripts of migration and invasion inhibitory protein (MIIP) are associated with increased survival in European-American patients with invasive breast carcinoma ${ }^{36}$.

We confirmed a consistent reduction of IR events in TCGA breast adenocarcinoma samples compared to adjacent normal breast tissue. While $\mathrm{BrCa}$ is the only cancer where this reduction is observed, IR frequencies are, in fact, comparable to those observed in other 
266

267

268

269

270

271

272

273

274

275

276

277

278

279

280

281

282

283

284

285

286

287

288

289

290

291

292

293

294

295

296

297

cancer types. This is due to the excessively large number of IR events in healthy breast tissue.

Gascard et al. found that IR increases with differentiation state in normal human breast cells with fewer IR events in myoepithelial cells and seven times more events in luminal epithelial cells ${ }^{37}$. Indeed, our results suggest that an important factor in the reduction of IR events in breast tumours is the changing cell composition from adipocyte and epithelial cell-rich breast tissue to lymphocyte-infiltrated breast tumours. Adipocytes and epithelial cells have one of the highest IR frequencies in their transcriptomes compared to other cell types, while lymphocytes are known to have low IR counts ${ }^{38}$. Siang and co-workers have shown in this context, that the RBP human antigen $\mathrm{R}(\mathrm{HuR})$, which is involved in pre-mRNA processing, is a negative regulator of adipogenesis ${ }^{39}$. Interestingly, Diaz-Muñoz et al. demonstrated that HuR binding to introns modulates alternative intron usage ${ }^{40}$. This may contribute to the high IR observed in adipocyte-rich normal breast tissue.

Aberrant IR has previously been associated with disease phenotypes and clinical outcomes. For example, IR in CMYC and SESTRIN1 genes was shown to be a reliable molecular marker separating melanoma from non-melanoma tumours ${ }^{14}$ and Sznajder and colleagues have shown that IR can be used as biomarker in hereditary repeat expansion diseases ${ }^{15}$. Despite marked differences between tumour and normal breast tissue, IR profiles in our analysis also differ between $\mathrm{ER}^{+}$vs $\mathrm{ER}^{-}$tumours. The survival advantages associated with high IR numbers in the Luminal B subtype suggest that this form of alternative splicing should be considered for therapeutic exploitation. However, the exact mechanisms whereby dynamic IR profiles lead to differences in clinical outcomes would be the subject of future studies.

The inverse relationship between IR and cell proliferation has been previously observed in the context of B-cell development and T-cell activation ${ }^{38,41}$. Our results demonstrate that the number of IR events positively correlates with longer cancer cell doubling times and that more IR events are associated with slower cell proliferation in BrCa. Our data shows that HER2 positive breast tumours have the lowest number of IR events. HER2 is known to induce cell proliferation in human cancers and is associated with poor prognosis in $\mathrm{BrCa}^{42}$. These results suggest that IR is a mechanism that counteracts tumour growth and would provide opportunities as therapeutic targets. Interestingly, the tumour suppressor Herstatin, expressed in healthy breast tissue ${ }^{43}$, is a splice variant of the oncogene HER2, with a retained intron $8^{44}$. Herstatin is a secreted autoinhibitor of Her $2^{44}$ and intron 8 retention is regulated by RBPs of the HNRNP1 family (including H1, D, and A2/B1) ${ }^{45}$. Koedoot and co-workers have 
298 demonstrated that inhibition of cell proliferation can be achieved via splicing factor

299 knockdown in $\mathrm{TNBC}^{46}$.

300 In summary, our study sheds light on the unique causes and consequences of aberrant

301 splicing in BrCa. The modulation of IR levels may offer novel opportunities for personalised

302 BrCa treatment, especially in hormone- and chemotherapy-resistant subtypes.

\section{Materials and methods}

\section{RNA-sequencing data/patient samples}

305 We retrieved data from nine tumour types and healthy adjacent tissue, including $615 \mathrm{BrCa}$

306 patient samples generated by the TCGA (Figure 1A). Only samples for which sequencing had 307 been performed at $>40 \mathrm{M}$ read depth were selected for analysis. Moreover, only tumour types 308 with at least 20 matched tumour/normal tissue samples where considered.

309 RNA-seq data were downloaded as BAM files using the R/Bioconductor package

310 TCGAbiolinks $^{47}$ and the command-line tool gdc-client v1.4.0 (github.com/NCI-GDC/gdc-

311 client) under an approved data access application. All files were checked for integrity.

312 Harmonized gene expression data in the form of HTseq counts ${ }^{48}$ were downloaded using

313 TCGAbiolinks ${ }^{47}$.

314 mRNA-sequencing and data analysis - MCF7 and MCF10A cells

315 Total RNA was isolated from MCF7 and MCF10A cells using Trizol (Invitrogen). The RNA 316 quality was assessed using RNA 6000 Nano Chips on an Agilent Bioanalyzer (Agilent 317 Technologies) to confirm an RNA integrity score of >7.0. mRNA-seq was performed by 318 Macrogen (Korea) using the Illumina Hi-Seq 2000 platform. RNA-seq libraries were 319 prepared from $>1 \mu \mathrm{g}$ of total RNA using TruSeq RNA sample prep kit (Illumina) according 320 to the manufacturers' instructions.

\section{Differential IR and gene expression analyses}

322 IR was quantified using IRFinder v1.2.0 ${ }^{17}$, using the Ensembl human genome (hg38, release 323 86) as reference. The IRFinder algorithm measures 20 parameters for IR detection in each 324 sample, including the median number of reads mapping to each nucleotide across the intron 325 length (intron depth, ID), the ratio of nucleotides within an intron with mapped reads 326 (coverage), the number of reads that map to the $5^{\prime}$ flanking exon and to another exon within 327 the same gene (splice left, SL), the number of reads that map to the $3^{\prime}$ flanking exon and to 
328 another exon within the same gene (splice right, SR), the number of reads spanning the exon-

329 exon junction (splice exact, SE) as well as the IR ratio:

$$
\frac{\mathrm{ID}}{\mathrm{ID}+\max (\mathrm{SL}, \mathrm{SR})}
$$

330 The following criteria were used for quantifying the number of IR events in a sample:

$$
\begin{aligned}
& 0.7 \leq \frac{\mathrm{SL}}{S R} \leq 1.3 \\
& (S L+S R)>10 \text { in } \geq 50 \% \text { of samples; } \\
& \text { coverage }>0.5 \text { in } \geq 50 \% \text { of samples; } \\
& \text { IR }>0.05 \text { in at } \geq 50 \% \text { normal or cancer samples }
\end{aligned}
$$

331 The number of IR events in a sample was determined based on introns with an IR ratio $>0.1$ 332 and meeting the filtering criteria described above. Introns not meeting these criteria were not 333 considered as being retained. Beta regression was used to identify differentially retained 334 introns (dIR) between cancer and adjacent normal tissues using the betareg $\mathrm{R}$ package ${ }^{49}$. 335 Since IR ratios are proportional data with values between 0 and 1, we reasoned that beta 336 regression was best suited to model IR and identify dIRs between normal and cancer tissues. 337 An absolute difference in the IR ratio $\left(\Delta \mathrm{IR}=\mathrm{IR}_{\text {Cancer }}-\mathrm{IR}_{\text {Nornal }}\right)$ of more than 0.1 with FDR338 adjusted $p<0.05$ was considered significant.

339 Dimensionality reduction, i.e. principal component analysis (PCA), of IR profiles was

340 performed using the package factoextra (github.com/kassambara/factoextra).

341 Differential gene expression between normal breast tissue and $\mathrm{BrCa}$ was performed using the 342 DESeq2 package ${ }^{50}$. Genes with an average read count $>10$ in all samples were selected for 343 differential gene expression analysis $(n=23,072)$. Genes with an absolute log 2 fold-change > 3441 and FDR-adjusted $p<0.05$ were considered significant. To identify genes that were 345 specifically differentially expressed in $\mathrm{BrCa}$, we removed genes that were differentially 346 expressed in any of the other 8 cancers and determined specificity by computing the z-score 347 on log-fold change using the log-fold change observed in $\mathrm{BrCa}$ as reference.

\section{Gene Ontology and RBP analyses}

349 Gene Ontology analysis was performed using the clusterProfiler package ${ }^{51}$. The false 350 discovery rate (FDR) approach was used for multiple testing correction. The list of 1,542 351 RBPs was taken from Gerstberger et $a .^{52}$. 


\section{Survival analysis}

353 Patient survival data was provided by the TCGA consortium. Survival analysis was

354 performed using packages Surv and survminer (github.com/kassambara/survminer).

\section{RNA binding protein motif detection}

356 RNA binding protein (RBP) motifs in position-weight matrix format (PWM) were retrieved

357 from the ATtRACT database (version $0.99 \beta)^{53}$, which contains 1,196 motifs corresponding

358 to 160 human RBPs. Sequences of $100 \mathrm{nt}$ were extracted from the regions flanking retained 359 and non-retained introns and scanned for the presence of motifs using the fimo tool provided 360 by the meme suite ${ }^{54}$.

\section{Acknowledgements}

363 The results shown here are based upon data generated by the TCGA Research 364 Network: https://www.cancer.gov/tcga. The authors acknowledge The University of Sydney 365 High Performance Computing service at The University of Sydney for providing resources 366 that have contributed to the research data reported within this paper.

367 Financial support was provided by National Health \& Medical Research Council Investigator 368 Grants (\#1177305, \#1196405) to J.E.J.R. and U.S. and Project Grants (\#507776, \#1128748)

369 to J.E.J.R. We also received support from the Cancer Council NSW (project grants RG11-12, 370 RG14-09, RG20-12 to J.E.J.R. and U.S.). M.M. is funded through a Victoria Cancer Agency 371 Fellowship.

\section{Authors' contributions}

373 J.S.S. and U.S. designed the research. J.S.S., U.S., and V.P. performed bioinformatic analyses 374 and interpreted the data. A.Y.M.A., J.J.L.W., and J.E.J.R. performed and/or supervised 375 experiments. J.S.S. and U.S. wrote the manuscript with help from M.M., J.E.V., J.J.L.W., and 376 J.E.J.R. All authors have read and agreed to the published version of the manuscript. 


\section{$378 \quad$ References}

3791 Mercer, T. R. et al. Targeted RNA sequencing reveals the deep complexity of the

380 human transcriptome. Nat Biotechnol 30, 99-104, doi:10.1038/nbt.2024 (2011).

3812 Schmitz, U. et al. Intron retention enhances gene regulatory complexity in vertebrates.

$382 \quad$ Genome Biol 18, 216, doi:10.1186/s13059-017-1339-3 (2017).

3833 Douglas, A. G. \& Wood, M. J. RNA splicing: disease and therapy. Brief Funct

$384 \quad$ Genomics 10, 151-164, doi:10.1093/bfgp/elr020 (2011).

3854 Monteuuis, G., Schmitz, U., Petrova, V., Kearney, P. S. \& Rasko, J. E. J. Holding on

386 to Junk Bonds: Intron Retention in Cancer and Therapy. Cancer Research 81, 779-

387

388

389

390

391

392

393

394

395

396

397

398

399

400

401

402

403

404

405

406

407

408

409

410 789, doi:10.1158/0008-5472.Can-20-1943 (2021).

5 El Marabti, E. \& Younis, I. The Cancer Spliceome: Reprograming of Alternative Splicing in Cancer. Front Mol Biosci 5, 80, doi:10.3389/fmolb.2018.00080 (2018).

6 Bonnal, S. C., Lopez-Oreja, I. \& Valcarcel, J. Roles and mechanisms of alternative splicing in cancer - implications for care. Nat Rev Clin Oncol 17, 457-474, doi:10.1038/s41571-020-0350-x (2020).

7 Fish, L. et al. Nuclear TARBP2 Drives Oncogenic Dysregulation of RNA Splicing and Decay. Mol Cell 75, 967-981 e969, doi:10.1016/j.molcel.2019.06.001 (2019).

8 Koh, C. M. et al. MYC regulates the core pre-mRNA splicing machinery as an essential step in lymphomagenesis. Nature 523, 96-100, doi:10.1038/nature14351 (2015).

9 Ziff, O. J. et al. Reactive astrocytes in ALS display diminished intron retention. Nucleic Acids Res 49, 3168-3184, doi:10.1093/nar/gkab115 (2021).

10 Schmitz, U. et al. Widespread Aberrant Alternative Splicing despite Molecular Remission in Chronic Myeloid Leukaemia Patients. Cancers (Basel) 12, doi:10.3390/cancers12123738 (2020).

11 Tang, A. D. et al. Full-length transcript characterization of SF3B1 mutation in chronic lymphocytic leukemia reveals downregulation of retained introns. Nat Commun 11, 1438, doi:10.1038/s41467-020-15171-6 (2020).

12 Smart, A. C. et al. Intron retention is a source of neoepitopes in cancer. Nat Biotechnol 36, 1056-1058, doi:10.1038/nbt.4239 (2018).

13 Lee, S. H. et al. Widespread intronic polyadenylation inactivates tumour suppressor genes in leukaemia. Nature 561, 127-131, doi:10.1038/s41586-018-0465-8 (2018).

14 Giannopoulou, A. F. et al. Gene-Specific Intron Retention Serves as Molecular Signature that Distinguishes Melanoma from Non-Melanoma Cancer Cells in Greek Patients. Int J Mol Sci 20, doi:10.3390/ijms20040937 (2019).

412

413

414

415

416

417

418 biomarker. Proc Natl Acad Sci U S A 115, 4234-4239, doi:10.1073/pnas.1716617115 (2018).

16 Seiler, M. et al. H3B-8800, an orally available small-molecule splicing modulator, induces lethality in spliceosome-mutant cancers. Nat Med 24, 497-504, doi:10.1038/nm.4493 (2018).

17 Middleton, R. et al. IRFinder: assessing the impact of intron retention on mammalian gene expression. Genome Biol 18, 51, doi:10.1186/s13059-017-1184-4 (2017).

18 Petrova, V. et al. Chromatin accessibility determines intron retention in a cell typespecific manner. bioRxiv, 2021.2002.2017.431609, doi:10.1101/2021.02.17.431609 (2021).

19 Monteuuis, G., Wong, J. J. L., Bailey, C. G., Schmitz, U. \& Rasko, J. E. J. The changing paradigm of intron retention: regulation, ramifications and recipes. Nucleic Acids Res 47, 11497-11513, doi:10.1093/nar/gkz1068 (2019). 
42720 Dvinge, H. \& Bradley, R. K. Widespread intron retention diversifies most cancer

428

429

430

431

432

433

434

435

436

437

438

439

440

441

442

443

444

445

446

447

448

449

450

451

452

453

454

455

456

457

458

459

460

461

462

463

464

465

466

467

468

469

470

471

472

473

474

475

476 transcriptomes. Genome Med 7, 45, doi:10.1186/s13073-015-0168-9 (2015).

21 Audic, S. \& Claverie, J. M. The significance of digital gene expression profiles. Genome Res 7, 986-995, doi:10.1101/gr.7.10.986 (1997).

22 Hudson, B. P., Martinez-Yamout, M. A., Dyson, H. J. \& Wright, P. E. Recognition of the mRNA AU-rich element by the zinc finger domain of TIS11d. Nat Struct Mol Biol 11, 257-264, doi:10.1038/nsmb738 (2004).

23 Lim, J. et al. Uridylation by TUT4 and TUT7 marks mRNA for degradation. Cell 159, 1365-1376, doi:10.1016/j.cell.2014.10.055 (2014).

24 Subramanian, A. et al. Gene set enrichment analysis: a knowledge-based approach for interpreting genome-wide expression profiles. Proc Natl Acad Sci U S A 102, 1554515550, doi:10.1073/pnas.0506580102 (2005).

25 Avila Cobos, F., Alquicira-Hernandez, J., Powell, J. E., Mestdagh, P. \& De Preter, K. Benchmarking of cell type deconvolution pipelines for transcriptomics data. Nat Commun 11, 5650, doi:10.1038/s41467-020-19015-1 (2020).

26 Aran, D., Hu, Z. \& Butte, A. J. xCell: digitally portraying the tissue cellular heterogeneity landscape. Genome Biol 18, 220, doi:10.1186/s13059-017-1349-1 (2017).

27 Wagner, J. et al. A single-cell atlas of the tumor and immune ecosystem of human breast cancer. Cell 177, 1330-1345. e1318 (2019).

28 Jackson, H. W. et al. The single-cell pathology landscape of breast cancer. Nature 578, 615-620 (2020).

29 Pal, B. et al. A single $\square$ cell RNA expression atlas of normal, preneoplastic and tumorigenic states in the human breast. The EMBO journal 40, e107333 (2021).

30 Braunschweig, U. et al. Widespread intron retention in mammals functionally tunes transcriptomes. Genome Res 24, 1774-1786, doi:10.1101/gr.177790.114 (2014).

31 Edwards, C. R. et al. A dynamic intron retention program in the mammalian megakaryocyte and erythrocyte lineages. Blood 127, e24-e34, doi:10.1182/blood2016-01-692764 (2016).

32 Green, I. D. et al. Macrophage development and activation involve coordinated intron retention in key inflammatory regulators. Nucleic Acids Res, doi:10.1093/nar/gkaa435 (2020).

33 Wong, J. J. et al. Orchestrated intron retention regulates normal granulocyte differentiation. Cell 154, 583-595, doi:10.1016/j.cell.2013.06.052 (2013).

34 Yeom, K. H. et al. Tracking pre-mRNA maturation across subcellular compartments identifies developmental gene regulation through intron retention and nuclear anchoring. Genome Res 31, 1106-1119, doi:10.1101/gr.273904.120 (2021).

35 Dvinge, H., Guenthoer, J., Porter, P. L. \& Bradley, R. K. RNA components of the spliceosome regulate tissue- and cancer-specific alternative splicing. Genome Res 29, 1591-1604, doi:10.1101/gr.246678.118 (2019).

36 Kim, D. et al. Population-dependent Intron Retention and DNA Methylation in Breast Cancer. Mol Cancer Res 16, 461-469, doi:10.1158/1541-7786.MCR-17-0227 (2018).

37 Gascard, P. et al. Epigenetic and transcriptional determinants of the human breast. Nat Commun 6, 6351, doi:10.1038/ncomms7351 (2015).

$38 \mathrm{Ni}$, T. et al. Global intron retention mediated gene regulation during CD4+ T cell activation. Nucleic Acids Res 44, 6817-6829, doi:10.1093/nar/gkw591 (2016).

39 Siang, D. T. C. et al. The RNA-binding protein HuR is a negative regulator in adipogenesis. Nat Commun 11, 213, doi:10.1038/s41467-019-14001-8 (2020).

40 Diaz-Munoz, M. D. et al. The RNA-binding protein HuR is essential for the B cell antibody response. Nat Immunol 16, 415-425, doi:10.1038/ni.3115 (2015). 
$47741 \quad$ Ullrich, S. \& Guigo, R. Dynamic changes in intron retention are tightly associated with regulation of splicing factors and proliferative activity during B-cell development. Nucleic Acids Res 48, 1327-1340, doi:10.1093/nar/gkz1180 (2020).

42 Iqbal, N. \& Iqbal, N. Human Epidermal Growth Factor Receptor 2 (HER2) in Cancers: Overexpression and Therapeutic Implications. Mol Biol Int 2014, 852748, doi:10.1155/2014/852748 (2014).

43 Koletsa, T. et al. A splice variant of HER2 corresponding to Herstatin is expressed in the noncancerous breast and in breast carcinomas. Neoplasia 10, 687-696, doi:10.1593/neo.08314 (2008).

44 Doherty, J. K., Bond, C., Jardim, A., Adelman, J. P. \& Clinton, G. M. The HER-2/neu receptor tyrosine kinase gene encodes a secreted autoinhibitor. Proc Natl Acad Sci U $S$ A 96, 10869-10874, doi:10.1073/pnas.96.19.10869 (1999).

45 Silipo, M., Gautrey, H., Satam, S., Lennard, T. \& Tyson-Capper, A. How is Herstatin, a tumor suppressor splice variant of the oncogene HER2, regulated? RNA Biol 14, 536-543, doi:10.1080/15476286.2016.1267074 (2017).

46 Koedoot, E. et al. Splicing factors control triple-negative breast cancer cell mitosis through SUN2 interaction and sororin intron retention. J Exp Clin Cancer Res 40, 82, doi:10.1186/s13046-021-01863-4 (2021).

47 Colaprico, A. et al. TCGAbiolinks: an R/Bioconductor package for integrative analysis of TCGA data. Nucleic Acids Res 44, e71, doi:10.1093/nar/gkv1507 (2016).

48 Anders, S., Pyl, P. T. \& Huber, W. HTSeq--a Python framework to work with highthroughput sequencing data. Bioinformatics 31, 166-169, doi:10.1093/bioinformatics/btu638 (2015).

Cribari-Neto, F. \& Zeileis, A. Beta Regression in R. Journal of Statistical Software 34, 1 - 24, doi:10.18637/jss.v034.i02 (2010).

50 Love, M. I., Huber, W. \& Anders, S. Moderated estimation of fold change and dispersion for RNA-seq data with DESeq2. Genome Biol 15, 550, doi:10.1186/s13059-014-0550-8 (2014).

51 Yu, G., Wang, L. G., Han, Y. \& He, Q. Y. clusterProfiler: an R package for comparing biological themes among gene clusters. OMICS 16, 284-287, doi:10.1089/omi.2011.0118 (2012).

52 Gerstberger, S., Hafner, M. \& Tuschl, T. A census of human RNA-binding proteins. Nat Rev Genet 15, 829-845, doi:10.1038/nrg3813 (2014).

53 Giudice, G., Sanchez-Cabo, F., Torroja, C. \& Lara-Pezzi, E. ATtRACT-a database of RNA-binding proteins and associated motifs. Database (Oxford) 2016, doi:10.1093/database/baw035 (2016). 